\title{
Development of a preliminary nomogram to predict progression of bone scan for castration- resistant prostate cancer
}

This article was published in the following Dove Press journal:

OncoTargets and Therapy

7 April 2015

Number of times this article has been viewed

\author{
Guo-Wen Lin ${ }^{1,2}$ \\ Ding-Wei $Y e^{1,2}$ \\ Hui-Xun Jia ${ }^{2,3}$ \\ Bo $\mathrm{Dai}^{1,2}$ \\ Hai-Liang Zhang ${ }^{1,2}$ \\ Yao Zhu', \\ Guo-Hai Shi ${ }^{1,2}$ \\ Chun-Guang $\mathrm{Ma}^{1,2}$ \\ 'Department of Urology, Fudan \\ University Shanghai Cancer Center, \\ ${ }^{2}$ Department of Oncology, Shanghai \\ Medical College, ${ }^{3}$ Department \\ of Clinical Statistics Center, Fudan \\ University Shanghai Cancer Center, \\ Fudan University, Shanghai, People's \\ Republic of China
}

\begin{abstract}
The optimal time to perform bone scan to detect new metastasis during the castration-resistant prostate cancer (CRPC) stage remains undefined. This study attempted to identify predictors of progression of bone scan for CRPC, and use such information to develop a nomogram to predict the optimal time of examinations for bone scan. The analysis included 167 CRPC patients. Progression of bone lesion, as evaluated by bone scan, occurred in 64 (38.3\%) cases. A logistic regression identified the following three risk factors: short time to prostate-specific antigen (PSA) progression, severe pain, and short PSA doubling time (PSADT) $(P<0.05$ for all). A nomogram model was constructed to predict progression of bone scan using time to PSA progression and severe pain as dichotomized variables and PSADT as a continuous variable. The result indicated that a predictive nomogram model showed a bootstrap-corrected concordance index of 0.762 and good calibration using the three readily available variables, and there were worse prognosis and higher progression rate of bone scan for patients with time to PSA progression $<6.6$ months, severe pain, and short PSADT ( $<2$ months). In conclusion, short time to PSA progression, severe pain, and short PSADT are three risk factors of progression of bone scan for CRPC patients. The predictive nomogram model may be a valuable numerical assessment tool for patient consultation and treatment decision.
\end{abstract}

Keywords: bone scan, castration-resistant prostate cancer, nomogram, predictor, progression

\section{Introduction}

Hormonal therapy is not a curative strategy for patients with advanced prostate cancer, although it remains one of its most important treatment options. Most patients eventually develop hormone-resistant disease with prostate-specific antigen (PSA) relapse and disease progression. Effective treatment options for castration-resistant prostate cancer (CRPC), unfortunately, are rather limited. ${ }^{1-4}$

One of the most common signs of disease progression in CRPC patients is new bone metastasis, the detection of which by bone scan allows urologists to make changes in treatment strategies. However, determination of the optimal time for a bone scan in the CRPC stage remains a challenging task for urologists. Inappropriate detection time may cause delays in treatment or failure in finding new lesions, while an undue frequency of detection may not be cost-effective. ${ }^{5}$ Currently, the optimal time for bone scan remains undefined, and there is an urgent need for inexpensive, convenient, and rapid predicting tools to assess the optimal time for performing bone scan.

To our knowledge, few studies have investigated reliable predicting tools to assess the optimal time of bone scan for CRPC patients. In this study, we focused on exploring predictors of progression of bone scan and constructing an individualized model to assess the optimal time to perform bone scan.
Correspondence: Ding-Wei Ye

Department of Urology, Fudan University Shanghai Cancer Center, 270 Dong'an

Road, Shanghai 200032, People's Republic of China

Tel +862164175590 ext I807

Fax +86 2I 64438640

Email dwyeli@I63.com 


\section{Materials and methods}

\section{Patients}

Five hundred thirty-eight patients with prostate adenocarcinoma sought treatment at Fudan University Cancer Center, Shanghai, People's Republic of China, between January 2011 and January 2013. Prostate adenocarcinoma was confirmed by prostate biopsy using the $6-12$ core strategy and staged using the tumor-node-metastasis (TNM) staging system according to the American Joint Committee on Cancer (AJCC) guideline based on biopsy and clinical or radiological evidence by computed tomography (CT), magnetic resonance (MR), and technetium Tc 99m methylene diphosphonate single-photon emission computed tomography coregistration with computed tomography (99m Tc-MDP SPECT/ CT). Of these patients, 167 cases were in the advanced stage (T3/T4 or N1 or M1) and had received androgen deprivation therapy (ADT), including orchiectomy or luteinizing hormone-releasing hormone agonists with an antiandrogen (flutamide or bicalutamide) as initial hormonal therapy. Patients were excluded if they had received prior radical treatment, corticosteroids, chemotherapy, aminoglutethimide or experimental therapy.

\section{Follow-up and assessment}

Patient plasma samples were collected monthly at the time of their visit for ADT at the cancer center. Symptoms were evaluated, physical examination was performed, and medical history was solicited at each visit, and imaging examination, including $\mathrm{CT}$, MR, and SPECT/CT, was made if disease progression was suspected.

CRPC was diagnosed according to the European Association of Urology Guidelines 2014 and was considered present if serum testosterone was $<50 \mathrm{ng} / \mathrm{dL}$ and there were three consecutive rises of PSA, at least 1 week apart, resulting in two $50 \%$ increases over the nadir, with a PSA level $>2 \mathrm{ng} / \mathrm{mL} .{ }^{6}$ All patients experienced the CRPC stage during the follow-up.

In this study, bone scan included MR of the vertebral column or limbs and SPECT/CT. Bone scan was advised because of rising PSA (97 cases) and/or mild/severe pain (145 cases) and/or elevating alkaline phosphatase (57 cases). Progression of bone scan was defined as progression or appearance of two or more new lesions compared to the bone scan at the initial ADT. The median duration of follow-up was 42.7 months (range: $18-60$ months).

\section{Statistical analysis}

The following variables were evaluated: age, clinical TNM stage, initial PSA, biopsy Gleason score, nadir PSA, time to PSA progression, the presence of pain, baseline PSA, alkaline phosphatase, and PSA doubling time (PSADT). Time to PSA progression was defined as the time from the initial ADT to a point in which there were at least a $25 \%$ increase in PSA over the nadir and an absolute increase of at least $2 \mathrm{ng} / \mathrm{mL}$. ${ }^{7}$ Pain was assessed using the Brief Pain Inventory Short Form, with a score of 0-2 representing no or mild pain and a score of 3-10 representing severe pain. ${ }^{8}$ The PSADT was calculated as the PSA kinetics over time from the nadir PSA to the baseline PSA, on the basis of the previous literature. ${ }^{9}$

Univariate and multivariate logistic regression analyses were used to test the association of clinicopathologic features with progression of bone scan. The restricted cubic splines (RCS) modeling could assist to estimate whether these clinicopathologic characteristics as categorical or continuous variables would optimize Akaike's information criterion (AIC) for the logistic regression analysis. ${ }^{10}$ The best cutoff of variables were evaluated by the Youden index, calculated as sensitivity + specificity $-1 .^{11,12}$ When the maximal index occurs at the cutoff, it optimizes the differentiating ability of the variable..$^{13}$

A nomogram model was constructed on the basis of the results of the multivariate logistic regression analysis, which was to predict the individual probability of progression of bone scan. For model validation, we assessed discrimination and calibration. Discrimination was measured using the $\mathrm{C}$-index, which is similar to the receiver operating characteristics (ROC) curve of the area under curve (AUC). ${ }^{10}$ Bootstrap-corrected C-indexes were used to better gauge expected future predictive accuracy. Calibration was assessed by visual inspection of the plots of predicted probability of progression vs actual outcome. $P<0.05$ was considered to be statistically significant. The RCS modeling and nomogram were established and analyzed by R 3.1.1, and other statistical calculations were computed by SPSS version 13.0 (SPSS Inc, Chicago, IL).

\section{Results}

\section{Patient demographic and baseline characteristics}

The demographic and clinicopathologic characteristics of the 167 enrolled patients are described in Table 1. Their median age was 70 years. The median initial PSA, nadir PSA, time to PSA progression, baseline PSA, baseline alkaline phosphatase, and PSADT were $120 \mathrm{ng} / \mathrm{mL}$, $0.46 \mathrm{ng} / \mathrm{mL}, 9.0$ months, $16.3 \mathrm{ng} / \mathrm{mL}, 94 \mathrm{IU} / \mathrm{L}$, and 1.87 months, respectively. According to the RCS modeling, the time to PSA progression and baseline alkaline phosphatase as categorical variables will optimize the AIC. 
Table I Patient demographic and baseline characteristics

\begin{tabular}{|c|c|c|c|}
\hline Characteristic & Progression of bone scan & No progression of bone scan & $P$-value* \\
\hline Age, years & & & $0.65 I$ \\
\hline Median (range) & $7 I(65-8 I)$ & $70(49-8 I)$ & \\
\hline Clinical T stage, n (\%) & & & 0.108 \\
\hline $\mathrm{T} 2$ & $47(4 I .6)$ & $66(58.4)$ & \\
\hline T3-T4 & $17(31.5)$ & $37(68.5)$ & \\
\hline Clinical N stage, n (\%) & & & $0.77 \mathrm{I}$ \\
\hline No & $4 I(39.0)$ & $64(61.0)$ & \\
\hline $\mathrm{NI}$ & $20(37.0)$ & $34(63.0)$ & \\
\hline Unknown & $3(37.5)$ & $5(62.5)$ & \\
\hline Clinical M stage, n (\%) & & & 0.112 \\
\hline Mo & $29(46.0)$ & $34(54.0)$ & \\
\hline MI & $35(33.7)$ & $69(66.3)$ & \\
\hline Biopsy Gleason score, n (\%) & & & 0.079 \\
\hline $4-6$ & $15(3 \mid .3)$ & $33(68.7)$ & \\
\hline 7 & $17(37.8)$ & $28(62.2)$ & \\
\hline $8-10$ & $28(45.2)$ & $34(54.8)$ & \\
\hline Unknown & $4(30.6)$ & $8(69.4)$ & \\
\hline Initial PSA, ng/mL & & & 0.058 \\
\hline Median (range) & $66(7-500)$ & $144(10-4,050)$ & \\
\hline Nadir PSA, ng/mL & & & 0.184 \\
\hline Median (range) & $0.4 \mathrm{I}(0.0 \mathrm{I}-18.43)$ & $0.50(0.03-69.42)$ & \\
\hline Time to PSA progression, $\mathrm{n}(\%)$ & & & $<0.001$ \\
\hline$<6.6$ months & $41(68.3)$ & $19(31.7)$ & \\
\hline$\geq 6.6$ months & $23(21.5)$ & $84(78.5)$ & \\
\hline Median (range) & $6.1(3.6-28.2)$ & $9.5(1.3-46.9)$ & \\
\hline Pain, n (\%) & & & $<0.001$ \\
\hline No or mild & $23(21.1)$ & $86(78.9)$ & \\
\hline Severe & $4 \mid(70.7)$ & $17(29.3)$ & \\
\hline Baseline alkaline phosphatase, n (\%) & & & 0.067 \\
\hline$<|30| \mathrm{IU} / \mathrm{L}$ & $56(56.0)$ & $44(44.0)$ & \\
\hline$\geq 130 \mathrm{IU} / \mathrm{L}$ & $47(70.1)$ & $20(29.9)$ & \\
\hline Median (range) & $87(56-435)$ & $97(53-6 \mid 2)$ & \\
\hline Baseline PSA, ng/mL & & & 0.014 \\
\hline Median (range) & $46.6(8.3-44.4)$ & $5.7(0.6-762)$ & \\
\hline PSADT, month & & & $<0.001$ \\
\hline Median (range) & $1.57(0.14-3.28)$ & $2.57(0.7 \mathrm{I}-33.67)$ & \\
\hline
\end{tabular}

Note: *P-value was calculated by the univariate logistic regression analysis. Abbreviations: PSA, prostate-specific antigen; PSADT, PSA doubling time.

At a cutoff of 6.6 months, time to PSA progression had a sensitivity of 0.704 and a specificity of 0.851 , with a maximal Youden index of 0.554 and the AUC of ROC of 0.777. Using a similar method, we chose 130 IU/L for baseline alkaline phosphatase.

\section{Predictors}

At the last follow-up, 38.3\% (64/167) cases showed progression of bone scan. The univariate logistic regression analysis revealed a significant difference in time to PSA progression, severe pain, baseline PSA, and PSADT (Table 1). Other variables were excluded from the final model because of their weak association with progression of bone scan $(P>0.5)$. The multivariate analysis further indicated that time to PSA progression, severe pain, and PSADT were markedly associated with, and were significant prognostic predictors of, progression of bone lesions (Table 2).

Of all the three predictors, pain (HR [hazards ratio] $=8.423$ ) represented the most informative predictor and the strongest determinant, followed by time to PSA progression $(\mathrm{HR}=4.079)$ and PSADT $(\mathrm{HR}=2.222)$. The progression rate of bone scan for patients with severe pain and no or mild pain were $70.7 \%(41 / 58)$ and $21.1 \%(23 / 109)$, respectively. The progression rate was $68.3 \%(41 / 60)$ for those with time to PSA progression $<6.6$ months, compared with $21.5 \%$ (23/107) for those with $\geq 6.6$ months. A shorter PSADT had a higher progression rate. Using the Youden index, we chose 2 months as the best cutoff for baseline PSA. Patients with PSADT $<2$ months had a progression rate of $57.0 \%(49 / 86)$ in comparison with $18.5 \%(15 / 81)$ for those with PSADT $\geq 2$ months. 
Table 2 Multivariate logistic regression analysis of progression of bone scan

\begin{tabular}{lllll}
\hline Variable & Status & HR* & HR 95\% Cl & P-value \\
\hline Pain & No/mild vs serious & 8.423 & $3.458-20.517$ & $<0.001$ \\
Time to PSA progression & $<6.6$ vs $\geq 6.6$ months & 4.079 & $1.750-9.507$ & 0.001 \\
PSADT & & 2.222 & $1.406-3.509$ & 0.001 \\
Baseline PSA & & 1.002 & $0.999-1.005$ & 0.208 \\
\hline
\end{tabular}

Notes: *When variables entered into the multivariate logistic regression analysis for progression of bone lesions. Hazards ratio (HR) of pain, time to PSA progression, and PSADT was the highest value in these variables, and $P<0.05$ only for these variables.

Abbreviations: $\mathrm{Cl}$, confidence interval; PSA, prostate-specific antigen; PSADT, PSA doubling time.

\section{A nomogram model}

The predicted model was presented as a nomogram showing the multivariate effects of the three factors (Figure 1). We further evaluated the discrimination and calibration of the nomogram. We found that the bootstrap-corrected C-index was 0.762 , indicating acceptable discrimination. The calibration plot further showed good calibration between predicted risk and observed incidence (Figure 2). According to the nomogram, the digital result from the nomogram was important for decision of a bone scan. If we chose the subgroups the most statistically significantly different (time to PSA progression $<6.6$ months, severe pain, and PSADT $<2$ months), the probability of progression of bone scan was $85 \%$ for patients with three risk factors, which was the optimal time to perform bone scan.

\section{Discussion}

Although introduced nearly a century ago, ADT still remains the top therapeutic choice for advanced prostate cancer patients, leading to improved quality of life and extended survival of these patients. However, the prognosis remains dismal for patients who have failed the initial ADT and whose disease has progressed to CRPC, and only limited therapeutic strategies are available for them. New drugs, such as ARN-509, abiraterone, enzalutamide, radium-223, and cabazitaxel, prolong survival only very modestly and cannot forestall disease progression. ${ }^{3,4,6,14}$ Bone metastases occur in more than $80 \%$ of cases with advanced-stage prostate cancer, which noticeably increase the risk of skeletalrelated events, jeopardizing the quality of life and increasing the incidence of comorbidities. Furthermore, patients with
Points

PSADT (month)

Time to PSA progression

Pain

Total points

$\begin{array}{llllllll}35 & 30 & 25 & 20 & 15 & 10 & 5 & 0\end{array}$

$\geq 6.6$ months

$<6.6$ months

No or mild pain

Predicting risk values for progression of bone scan

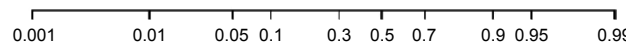

Figure I Nomogram to predict progression of bone scan after patients entered CRPC stage.

Notes: A nomogram is constructed based on 167 advanced prostate cancer patients to predict progression of bone scan after the patients have entered the CRPC stage. PSA ( $\mathrm{ng} / \mathrm{mL}$ ). To obtain the nomogram-predicted probability of progression, we locate values of a patient at each axis and draw a vertical line to the "Points" axis to determine how many points are attributed to each variable value. The total points for all variables are calculated, and the sum on the "Total points" line is then located to assess the individual probability on the predicting risk values for progression of bone scan.

Abbreviations: CRPC, castration-resistant prostate cancer; PSA, prostate-specific antigen; PSADT, PSA doubling time. 


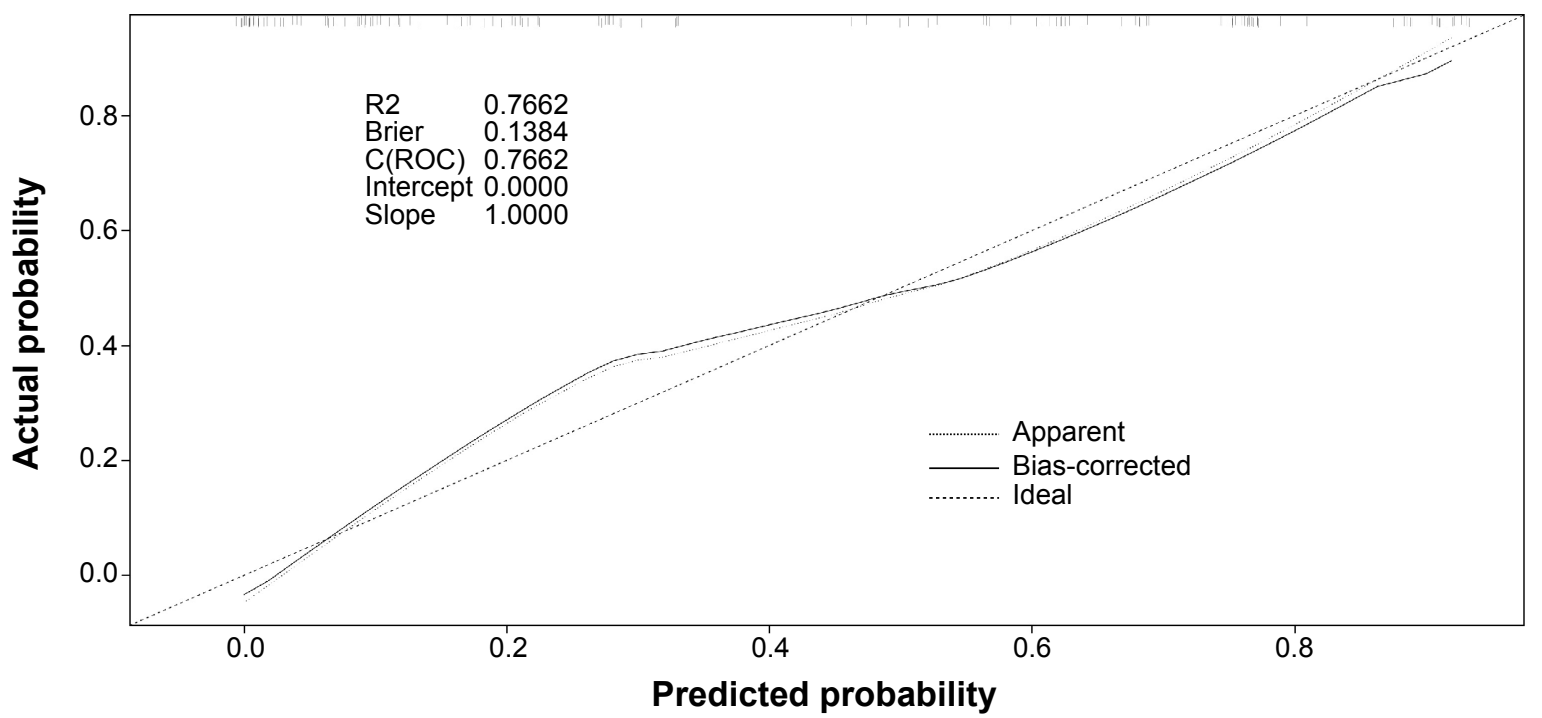

Figure 2 Calibration plot of the predicted and observed probabilities of progression of bone scan in the study cohort ( $n=167)$.

Notes: The calibration plot shows the performance of the nomogram. The $x$-axis shows the prediction calculated using the nomogram, and the observed rate of progression is plotted on the $y$-axis. Perfect prediction would correspond to a slope of I (diagonal $45^{\circ}$ broken line). The solid line indicates the bootstrap-corrected nomogram performance according to internal validation cohort.

bone metastases have a 5-year survival rate of merely $25 \%$, with a median overall survival (OS) of approximately 40 months. ${ }^{15,16}$

SPECT/CT and MR are the most common diagnostic methods for bone metastases. A considerable proportion of patients are advised to undergo bone scan after entering the CRPC stage; however, the key issue is the indeterminate nature of the optimal bone scan time in clinical practice. A late bone scan unnecessarily delays treatment of patients, whereas a premature bone scan fails to reveal the relevant evidence of progression. On the other hand, frequent bone scans inevitably increase the financial burden of the patients, and ambiguities in bone scan findings adversely impact the diagnosis and treatment strategy. ${ }^{17}$ Therefore, an informed approach to the determination of the optimal bone scan time remains a critical issue clinically. If highly informative predictors of progression of bone scan are identified and validated, especially for individualized prediction, an informed decision can then be made for the optimal time of bone scan.

This study sought to delineate meaningful predictors of progression of bone scan by examining common clinicopathologic variables, and we found that pain severity, time to PSA progression, and PSADT could predict progression of bone scan. Using these three predictors, we successfully constructed an individualized predictive nomogram model. PSA originates from prostatic cells and reflects the tumor cell burden, ${ }^{18}$ which is a specific diagnostic marker for prostate cancer and can be used for monitoring disease activities. ${ }^{19}$ We found that PSADT was one of the strongest determinants of progression of bone scan. This observation is consistent with the findings in existing literature. Sourbeer et $\mathrm{al}^{20}$ indicated that patients with shorter PSADT were more likely to receive a second bone scan. According to Brockman et $\mathrm{al}^{21}$ a PSADT in a nomogram based on standard parameters modestly improved predictive accuracy for biochemical recurrence after radical prostatectomy.

Our study showed that time to PSA progression at a cutoff of 6.6 months provided a maximal Youden index and was capable of differentiating progression risks of patients. An equally plausible explanation is that time to PSA progression reflects sensitivity of prostate cancer to ADT, with a longer time to PSA progression indicating a higher sensitivity to ADT and hence a more favorable prognosis. Time to PSA progression has been shown to be a reliable predictor of prognosis and heralds clinical progression. ${ }^{22}$ Hussain et $\mathrm{al}^{22}$ showed that the median OS was 10 months for CRPC patients with time to PSA progression $<7$ months, compared with 44 months for CRPC patients whose time to PSA progression was $>7$ months.

We speculated that, as a manifestation of bone destruction, severe pain was an adverse predictor of survival. In this study, we found that 58 cases $(34.7 \%)$ had severe pain with a significantly higher progression rate of bone scan (70.7\%) than did patients with mild or no pain (21.1\%). Our observation is consistent with those of other investigators. Gater et $\mathrm{al}^{8}$ showed that bone pain is a predictor of patient prognosis, while Armstrong et $\mathrm{al}^{23}$ identified and validated 
pain as a predictor of PSA decline and OS. Our data and other studies together suggest that pain severity may correlate with progression of bone scan.

Furthermore, using the three factors we constructed a predictive nomogram model, which allows stratification of progression risks and provides a numerical estimate to help clinicians and patients weigh the pros and cons of the decision of a bone scan. Using the predictive model, a patient with three predicted factors would be confronted with a probable progression rate of more than $85 \%$ and a HR of 4.6 compared to patients without any of the three factors. The data suggest that the optimal time to perform bone scan is when the three risk factors are present in a patient.

We noted that the Gleason score, TNM stage, initial PSA, nadir PSA, and baseline alkaline phosphatase showed no association with progression of bone scan in our cohort. In fact,

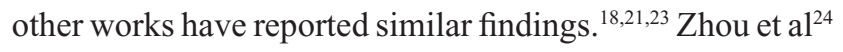
found that alkaline phosphatase was not correlated with bone metastasis. Furthermore, the long interval from primary diagnosis to the CRPC stage and various intricate prior treatments also affect the predictive power of various parameters. The limitations of our study include its single-center data and limited sample size, which may be responsible for the bias-corrected calibration curve being not completely parallel with the ideal curve. Furthermore, the predictive accuracy of the nomogram should be tested in an independent cohort, which represents the gold standard to confirm the validity of prediction models.

\section{Conclusion}

Short time to PSA progression, severe pain, and short PSADT are three risk factors of progression of bone scan for CRPC patients. The nomogram model as a predictive tool would be useful for patient consultation and urologist decision making.

\section{Acknowledgment}

This project was supported by the Shanghai Health Bureau of Research Fund (20144Y0180).

\section{Author contributions}

DWY carried out the conception and design, and along with all other authors contributed toward data analysis, drafting, and critically revising the paper. All authors agree to be accountable for all aspects of the work.

\section{Disclosure}

The authors report no conflicts of interest in this work.

\section{References}

1. Nakabayashi M, Oh WK, Jacobus S, et al. Activity of ketoconazole after taxane-based chemotherapy in castration-resistant prostate cancer. BJU Int. 2010;105:1392-1396.

2. Suzuki H, Okihara K, Miyake H, et al. Alternative nonsteroidal antiandrogen therapy for advanced prostate cancer that relapsed after initial maximum androgen blockade. J Urol. 2008;180: 921-927.

3. de Bono JS, Oudard S, Ozguroglu M, et al. Prednisone plus cabazitaxel or mitoxantrone for metastatic castration-resistant prostate cancer progressing after docetaxel treatment: a randomised open-label trial. Lancet. 2010;376:1147-1154.

4. de Bono JS, Logothetis CJ, Molina A, et al. Abiraterone and increased survival in metastatic prostate cancer. N Engl J Med. 2011;364: 1995-2005.

5. Taneja SS. Imaging in the diagnosis and management of prostate cancer. Rev Urol. 2004;6:101-113.

6. Heidenreich A, Bastian PJ, Bellmunt J, et al. EAU guidelines on prostate cancer. Part II: Treatment of advanced, relapsing, and castrationresistant prostate cancer. Eur Urol. 2014;65:467-479.

7. Scher HI, Halabi S, Tannock I, et al. Design and end points of clinical trials for patients with progressive prostate cancer and castrate levels of testosterone: recommendations of the Prostate Cancer Clinical Trials Working Group. J Clin Oncol. 2008;26:1148-1159.

8. Gater A, Abetz-Webb L, Battersby C, et al. Pain in castration-resistant prostate cancer with bone metastases: a qualitative study. Health Qual Life Outcomes. 2011;9:88.

9. Lin GW, Yao XD, Ye DW, et al. The prognostic factors of ketoconazole effective treatment for metastatic castration resistant prostate cancer: who can benefit from ketoconazole therapy? Asian J Androl. 2012;14(5):732-737.

10. Zhu Y, Zhang HL, Yao XD, et al. Development and evaluation of a nomogram to predict inguinal lymph node metastasis in patients with penile cancer and clinically negative lymph nodes. $J$ Urol. 2010;184: 539-545.

11. Schisterman EF, Perkins NJ, Liu A, Bondell H. Optimal cut-point and its corresponding Youden Index to discriminate individuals using pooled blood samples. Epidemiology. 2005;16:73-81.

12. Ruopp MD, Perkins NJ, Whitcomb BW, Schisterman EF. Youden Index and optimal cut-point estimated from observations affected by a lower limit of detection. Biom J. 2008;50:419-430.

13. Zang RY, Harter P, Chi DS, et al. Predictors of survival in patients with recurrent ovarian cancer undergoing secondary cytoreductive surgery based on the pooled analysis of an international collaborative cohort. Br J Cancer. 2011;105:890-896.

14. Rathkopf DE, Morris MJ, Fox JJ, et al. Phase I study of ARN-509, a novel antiandrogen, in the treatment of castration-resistant prostate cancer. J Clin Oncol. 2013;31:3525-3530.

15. Sturge J, Caley MP, Waxman J. Bone metastasis in prostate cancer: emerging therapeutic strategies. Nat Rev Clin Oncol. 2011;8: 357-368.

16. Jin JK, Dayyani F, Gallick GE. Steps in prostate cancer progression that lead to bone metastasis. Int $J$ Cancer. 2011;128:2545-2561.

17. Dotan ZA, Bianco FJ, Rabbani F, et al. Pattern of prostate-specific antigen (PSA) failure dictates the probability of a positive bone scan in patients with an increasing PSA after radical prostatectomy. $J$ Clin Oncol. 2005;23:1962-1968.

18. Ozu C, Nakashima J, Horiguchi Y, Oya M, Ohigashi T, Murai M. Prediction of bone metastases by combination of tartrate-resistant acid phosphatase, alkaline phosphatase and prostate specific antigen in patients with prostate cancer. Int J Urol. 2008;15:419-422.

19. Scher HI, Morris MJ, Basch E, Heller G. End points and outcomes in castration-resistant prostate cancer: from clinical trials to clinical practice. J Clin Oncol. 2011;29:3695-3704.

20. Sourbeer KN, Howard LE, Moreira DM, et al. Practice patterns and predictors of follow-up imaging after a negative bone scan in men with CRPC: results from the SEARCH database. J Urol. 2014;5347(14): $4857-4858$ 
21. Brockman JA, Alanee S, Vickers AJ, et al. Nomogram predicting prostate cancer-specific mortality for men with biochemical recurrence after radical prostatectomy. Eur Urol. 2014;2838(14):956-957.

22. Hussain M, Goldman B, Tangen C, et al. Prostate-specific antigen progression predicts overall survival in patients with metastatic prostate cancer: data from Southwest Oncology Group Trials 9346 (Intergroup Study 0162) and 9916. J Clin Oncol. 2009;27:2450-2456.
23. Armstrong AJ, Tannock IF, de WitR, George DJ, Eisenberger M, Halabi S. The development of risk groups in men with metastatic castrationresistant prostate cancer based on risk factors for PSA decline and survival. Eur J Cancer. 2010;46:517-525.

24. Zhou JQ, Zhu Y, Yao XD, et al. [The building and validation of a model to predict the bone metastases of patients with newly diagnosed prostate cancer]. Zhonghua Wai Ke Za Zhi. 2012;50:999-1002. Chinese.

\section{Publish your work in this journal}

OncoTargets and Therapy is an international, peer-reviewed, open access journal focusing on the pathological basis of all cancers, potential targets for therapy and treatment protocols employed to improve the management of cancer patients. The journal also focuses on the impact of management programs and new therapeutic agents and protocols on

\section{Dovepress}

patient perspectives such as quality of life, adherence and satisfaction. The manuscript management system is completely online and includes a very quick and fair peer-review system, which is all easy to use. Visit http://www.dovepress.com/testimonials.php to read real quotes from published authors.

Submit your manuscript here: http://www.dovepress.com/oncotargets-and-therapy-journal 